\title{
RENDIMIENTO Y ATRIBUTOS DE CALIDAD DE MORA (Rubus glaucus Benth) DE CUATRO ZONAS PRODUCTORAS DE BOLÍVAR
}

\author{
YIELD AND QUALITY ATTRIBUTES OF BLACKBERRY (Rubus glaucus Benth) OF \\ FOUR PRODUCING ZONES OF BOLÍVAR.
}

\footnotetext{
Rubén Saltos ${ }^{(1)}$, Martha González ${ }^{(2)}$, Víctor González ${ }^{(3)}$, Fernando Cofre ${ }^{(3)}$, Irene Hidalgo ${ }^{(3)}$, Lucia García ${ }^{(3)}$, Edwin Borja ${ }^{(4)}$.

(I) Ministerio de Agricultura y Ganadería. Guaranda-Ecuador.E-mail: rsaltos@mag.gob.ec (2) Universidad Estatal de Bolivar, "Alpachaca” Av. Ernesto Che Guevara s/n y Av. Gabriel Secaira. Guaranda-Ecuador.

(3) Universidad Estatal Amazónica, Km. 2. 1/2 vía Puyo a Tena (Paso Lateral). Puyo - Ecuador.

(4) Instituto Nacional de Investigaciones Agropecuarias INIAP. Email: bbenkas@yahoo.com https://doi.org/10.33789/talentos.7.2.133
}

\begin{abstract}
Resumen: Los atributos de calidad son instrumentos de medición que garantizan la calidad de los productos generando oportunidades de mercado. Sin embargo, varias actividades en el manejo, cosechay poscosech a son realizadas de manera empírica afectando la calidad de la frutayel sustento económico de pequeños y medianos productores. En esta investigación se evaluaron los siguientes parámetros: calibre y peso de frutos, estado de madurez, rendimiento de la pulpa, sólidos totales solubles, $\mathrm{pH}$, acidez titulable e índice de madurez. Los frutos cosechados dentro de la clasificación como calibre pequeño y pesos ( $\leq 6 g$ según la norma NTE-INEN 2427) representaron el mayor porcentaje (77,19 al 94,44\%) en las zonas de estudio; el mayor rendimiento de pulpa (71,48\%) se obtuvo en la zona de Guantug Cruz, con frutos que se concentran en estado de madurez en la categoría de 5 a 6, pH de 2,66 a 2,79, sólidos totales solubles de 6,5 a 7,6 ${ }^{\circ}$ Brix, acidez titulable de 2,56 a 3,02\% y con índices de madurez de 2,43 a 2,97. Los atributos acidez titulable, sólidos totales solubles y el índice de madurez no cumplen con el marco normativo estándar del Ecuador, esto es una limitante para la conservación de la fruta y alargar la vida útil sin procesamiento.
\end{abstract}

Palabras clave: Acidez titulable, indice de madurez, rendimiento de pulpa, sólidos totales solubles, cosecha.

Recibido: 29 de marzo de 2020

Aceptado: 01 de octubre de 2020

Publicado como artículo científico en la Revista de Investigación Talentos VII (2), 33-45 
Abstract: Quality attributes are measurement instruments that guarantee the quality of products, generating market opportunities. However, various activities in handling, harvesting and postharvesting are carried out empirically, affecting the quality of the fruit and the economic sustenance of small and medium producers. In this investigation the following parameters were evaluated: fruit size and weight, state of maturity, pulp yield, total soluble solids, $\mathrm{pH}$, titratable acidity and maturity index. The fruits harvested within the classification as small size and weights $(\leq 6 g$ according to the NTE-INEN 2427 standard) represented the highest percentage (77,19 to 94,44\%) in the study areas; the highest pulp yield (71,48\%) was obtained in the Guantug Cruz area, with fruits that are concentrated in a state of maturity in the category of 5 to $6, \mathrm{pH}$ of 2,66 to 2,79, total soluble solids from 6,5 to 7,6 ${ }^{\circ}$ Brix, titratable acidity from 2,56 to 3,02\% and with maturity indexes of 2,43 to 2,97. The attributes titratable acidity, total soluble solids and the maturity index do not comply with the Ecuadorian standard regulatory framework, this is a limitation for the conservation of the fruit and to extend the shelf life without processing.

Keywords: Titratable acidity, maturity index, pulp yield, total soluble solids, harvest.

\section{INTRODUCCIÓN}

La mora (Rubus glaucus Benth) es una especie nativa de las zonas tropicales altas de América (Franco y Giraldo, 1999), muy popular y ampliamente cultivado en todo el mundo (Türemis et al., 2003). En el año 2005 se estimó que la producción mundial alcanzo $154.644 \mathrm{t}$ cosechadas en 20.035 ha, un $45 \%$ más que en 1995 (Strik et al., 2007). En el 2008 se cultivaron 25.000 ha (Strik et al., 2008). Actualmente, el mayor productor mundial es Estados Unidos, seguido de México y China.

Esta especie en los Andes crece durante todo el año (Martínez et al., 2007), cultivada tradicionalmente por pequeños productores, con alto potencial de producción (MorenoMedina et al., 2016), incrementándose rápidamente en las últimas décadas (Strik et al., 2007; Meyers et al., 2014). En América Latina la mora se encuentra distribuida en las zonas altas tropicales principalmente de Colombia, Ecuador, Chile, Panamá, Guatemala, Honduras, México y El Salvador (PAVUC, 2007).
Para el año 2015, en Ecuador la superficie de mora cultivada alcanzó 5.048 ha, con una productividad de 6,80 t/ha (INIAP, 2016; en Barrera et al., 2017), distribuida a lo largo del callejón Interandino (Martínez et al., 2007), siendo la provincia Bolívar la mayor productora con 13.210 t/año equivalente al $39 \%$ de la producción nacional de la fruta, con una extensión de 1.902 ha y un rendimiento de 6,90 t/ha (Barrera et al., 2017), permitiendo el sustento económico permanente de pequeños y medianos productores en los cantones Chillanes, San Miguel, Chimbo y Guaranda.

El fruto es una baya elipsoidal, formada por pequeñas drupas, de color verde cuando se forma, pasando a rojo y luego a morado oscuro y brillante (Universidad de Antioquia, 2009). El color de las bayas varia conforme van madurando (Morales et al., 1996); se caracterizan por ser no climatéricos, altamente perecederos, con una vida en anaquel de 3 a 5 días (Freire, 2012).Su peso varía entre 4 a $11 \mathrm{~g} /$ fruta, 1 a $2 \mathrm{~cm}$ de diámetro, con 10 a $11^{\circ}$ Brix (Vasco et al., 2008). Son una fuente 
potencial de polifenoles (Mertz et al., 2007), contienen aproximadamente $35 \mathrm{mg} / \mathrm{g}$ de fruta (peso seco) de antocianina (Bridgers et al., 2010) y cerca del $90 \%$ de su contenido es agua (Sora et al., 2006).

La madurez de la fruta en la cosecha es uno de los principales factores que contribuyen y determinan su calidad (Perkins-Veazie et al., 1999). Estos deben cosecharse generalmente cuando presentan un color púrpura oscuro/negro brillante y una firmeza óptima (Bejarano, 1992; citado por Horvitz et al., 2017), en esta etapa, también se obtiene el mejor sabor (Skrovankova et al., 2015). Por su estructura morfológica frágil, enfrenta continuos cambios fisicoquímicos y de firmeza, muy susceptible a la pérdida de agua, ablandamiento y lesiones mecánicas que afectan su calidad (Junqueira-Gonçalves et al., 2016).

El manejo inadecuado durante las operaciones de precosecha, cosecha, selección, empaque, transporte, conservación y comercialización; afecta su calidad provocando la generación de lixiviados en su venta, deformación, pérdida de pigmentación, fermentación $\mathrm{y}$ proliferación de hongos (Ayala et al., 2013), como moho gris, causada por Botrytis cinerea y pudrición por Rhizopus stolonifer (Junqueira-Gonçalves et al., 2016) causando pérdidas de aproximadamente el $60 \%$ de su producción (Sora et al., 2006).

La heterogeneidad de la fruta, envases de recolección, tamaño de la fruta, formas de empaque, almacenamiento $y$ transporte durante de cosecha y poscosecha conllevan problemas en los volúmenes ofertados y la aceptación en el mercado, causando pérdidas económicas, limitando la competitividad en el mercado nacional e internacional. Los atributos de calidad evaluados serán considerados a corto y mediano plazo para el mejoramiento de las actividades en la cosecha y comercialización por parte de los productores, con mejor calidad del producto expedido hacia el consumidor local generando mayor ingreso económico a los productores.

\section{MATERIALES Y MÉTODOS}

\section{A. Área de Estudio}

La Provincia de Bolívar se encuentra en la Zona Centro de la Región Interandina del Ecuador, al occidente de la cordillera Occidental, en la zona de transición entre las regiones Litoral y Sierra, por esta razón goza de una gran variedad de climas, frio en los páramos y cálido en la zona subtropical, las temperaturas varían según la altitud, entre 12 y $16{ }^{\circ} \mathrm{C}$. La precipitación fluctúa entre 750 y $3000 \mathrm{~mm}$ (figura 1). Las características geográficas y climáticas de las cuatro zonas de estudio se representan en la tabla 1.

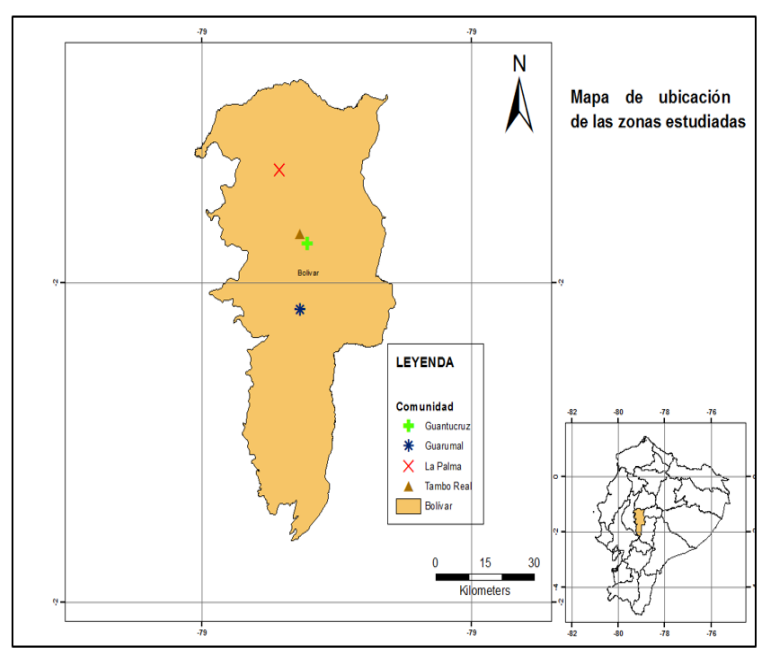

Fig. 1. Mapa de ubicación de cuatro zonas productoras de $R$. glaucus estudiadas en la provincia Bolívar. 
Revista de Investigación Talentos, Volumen VII (2), Julio - Diciembre 2020 ISSN Impreso: 1390-8197 ISSN Digital: 2631-2476

Tabla I. CARACTERÍSTICAS GEOGRÁFICAS Y CLIMÁTICAS DE LAS ZONAS DE ESTUDIO

\begin{tabular}{|c|c|c|c|c|c|c|c|}
\hline \multirow[t]{2}{*}{ Cantón } & \multirow[t]{2}{*}{ Parroquia } & \multirow[t]{2}{*}{ Comunidad } & \multicolumn{2}{|c|}{$\begin{array}{l}\text { Coordenadas } \\
\text { Geográficas }\end{array}$} & \multirow{2}{*}{$\begin{array}{c}\text { Altitud } \\
\text { (m.s.n.m.) }\end{array}$} & \multirow{2}{*}{$\begin{array}{l}\text { Precipitación } \\
\text { (mm anuales) }\end{array}$} & \multirow{2}{*}{$\begin{array}{c}\text { Temperatura } \\
\text { promedio } \\
\left({ }^{\circ} \mathrm{C}\right)\end{array}$} \\
\hline & & & Latitud & Longitud & & & \\
\hline & & & & & & 750 a 1750 & \\
\hline Guaranda & Guanujo & Tambo Real & 0711263 & 9834115 & 2319 & $\begin{array}{l}\text { Centro oeste } \\
\text { de Guaranda }\end{array}$ & 12 a 16 \\
\hline Guaranda & Guanujo & $\begin{array}{c}\text { Guantug } \\
\text { Cruz }\end{array}$ & 0713499 & 9831865 & 2595 & $\begin{array}{c}750 \text { a } 1750 \\
\text { Centro oeste } \\
\text { de Guaranda }\end{array}$ & 12 a 16 \\
\hline Guaranda & Salinas & La Palma & 0705109 & 9850444 & 2004 & $\begin{array}{c}2000 \text { a } 3000 \\
\text { Oeste de la } \\
\text { parroquia } \\
\text { Salinas }\end{array}$ & 12 a 16 \\
\hline Chimbo & Magdalena & Guarumal & 0711223 & 9814999 & 2460 & $750-1000$ & 14 a 16 \\
\hline
\end{tabular}

Fuente. Plan de Desarrollo y Ordenamiento Territorial, 2018.

\section{B. Material de estudio}

Se colectaron muestras de mora (frutos) de 10 recipientes de plástico de $15 \mathrm{~L}$ (balde) al azar de los productores de cuatro zonas de estudio, posteriormente fueron colocadas en tarrinas de Ptereftalato de Polietileno (PET), para su análisis en el Laboratorio de Alimentos de la Universidad Estatal de Bolívar.

\section{Métodos}

Los atributos de calidad se evaluaron considerando la Norma Técnica Ecuatoriana del Instituto Ecuatoriano de Normalización [INEN], (2015), en base al porcentaje de acidez titulable (ácido cítrico), máximo de 1,8 (NTE INEN 381, 2010), sólidos solubles totales expresados en ${ }^{\circ}$ Brix mínimo de 9,0 y el índice de madurez con 5,0 como mínimo (NTE INEN 380, 2015), resultante de la relación simple entre ${ }^{\circ}$ Brix y acidez titulable.

\section{C.1 Calibre de los frutos ( $\mathrm{mm})$}

La clasificación de los frutos se realizó en función de sus calibres pequeño, mediano y grande, (INEN, 2015). Como se muestra en la tabla 2 .

TABLA II. CALIBRE DE LA FRUTA: MORA DE CASTILLA

\begin{tabular}{ccc}
\hline Calibre & Diámetro en mm & Longitud en mm \\
\hline Grande & $>25$ & $>25$ \\
Mediano & $25-18$ & $25-20$ \\
Pequeño & $<18$ & $<20$ \\
\hline
\end{tabular}

Fuente: NTE INEN 2427, 2015

El efecto de esta variable se determinó mediante un ANOVA unifactoral, en el programa estadístico Statistical Package for the Social Sciences-SPSS versión 19.0 (Castañeda et al., 2010, citado por Saltos, R., 2019). Las diferencias entre calibres de la fruta se obtuvieron usando la prueba post hoc del test de Tukey al 5\% (Zar, 2010; Sokal y Rohlf, 2012). 


\section{C.2 Peso de los frutos}

En una balanza digital se pesaron los frutos para determinar la frecuencia absoluta de los pesos, para ello se establecieron rangos de peso promedio para la clasificación en función de su calibre, es así que se clasifico en: pequeño (peso menor o igual a $6 \mathrm{~g}$ ), calibre mediano (peso entre 6,1-8,6g) y calibre grande (peso mayor a $8,7 \mathrm{~g}$ ).

\section{C.3 Estado de madurez.}

La evaluación del color se realizó de forma visual considerando 6 estados de madurez, como se muestra en la figura 2 , en base a los requisitos de la Normativa técnica Ecuatoriana INEN 2427 (2010).

\section{C.4 Rendimiento de la pulpa}

Posterior a la obtención del peso de los frutos $(100 \mathrm{~g})$ de cada muestra, se realizó un escaldado por un tiempo de aproximadamente 5 minutos hasta ablandar los tejidos de las moras. Posteriormente, se despulpó el producto con ayuda de un extractor de jugos, se refinó una vez, se pesó la pulpa obtenida y se determinó el rendimiento de la pulpa mediante el uso de la siguiente ecuación:

$\%$ pulpa $\frac{P p}{P f} * 100$

Dónde:

$P f=$ Peso de la fruta entera

$P p=$ Peso de la pulpa
C.5 Sólidos solubles

Se colocaron dos gotas de la pulpa de la fruta sobre el prisma de la superficie de un refractómetro manual y se tomó la medida en grados Brix (Asociación de Químicos Analíticos Oficiales, [A.O.A.C], 2007).

\section{C.6 pH}

Se empleó un potenciómetro de electrodo de membrana cilíndrica previamente calibrado con buffer a pH 7 y pH 4. Se tomaron $20 \mathrm{ml}$ de pulpa de mora, se introdujo el electrodo en el centro de la muestra con agitación constante y se registró la lectura una vez estabilizado el valor, según el método escrito por la A.O.A.C. (2007).

\section{C.7 Acidez titulable}

Se pesaron $30 \mathrm{~g}$ de pulpa y se diluyeron a 200 $\mathrm{ml}$ con agua destilada. Se tomó una alícuota de $20 \mathrm{ml}$ y se tituló con hidróxido de sodio 0,1 $\mathrm{N}$ estandarizado, hasta el pH de 8,2 que es el punto de viraje del indicador fenolfatleína. Se registró la lectura del gasto, según lo descrito por la A.O.A.C. (2007).

Se calculó la acidez titulable con la siguiente ecuación:

Ácidez titulable (\% ácido cítrico) $=\frac{V_{N A O H} \times N \times m e q \times V t}{P m \times V a} \times 100$

Donde:

$\mathrm{V}_{\mathrm{NaOH}}=$ Volumen de hidróxido de sodio consumidos en la titulación $(\mathrm{mL})$

$\mathrm{N}=$ Normalidad del hidróxido de sodio 
meq $=$ Miliequivalentes del ácido cítrico 0,064

$\mathrm{Vt}=$ Volumen final $(\mathrm{ml})$

$\mathrm{Pm}=$ Peso de la muestra $(\mathrm{g})$

$\mathrm{Va}=$ Volumen de la alícuota $(\mathrm{ml})$

\section{C.8 Índice de madurez}

Se determinó el índice de madurez con la siguiente ecuación:

Índice de madurez $=\frac{\text { Sólidos Solubles Totales }\left({ }^{\circ} \text { Brix }\right)}{\text { Acidez } \text { Titulable }(\% \text { Ác.citrico })} \times 1$

\section{RESULTADOS Y DISCUSIÓN}

\section{A. Calibre y peso de frutos}

En la Tabla 3, se registraron los calibres y pesos de los frutos recolectados, el calibre pequeño (peso menor o igual a $6 \mathrm{~g}$ ) en las zonas productoras evaluadas presentó los valores más altos ( $\square=87,06 \%$ ). Estos valores son similares a los descritos por Horvitz et al., (2017), quienes indicaron en su estudio, que el tamaño de fruto aumentó con la maduración, el fruto cosechado en la etapa 5 alcanzó un peso promedio de 6,25 $\pm 1,49 \mathrm{~g}$ y $21,8 \pm 0,22$ y $26,6 \pm 0,32 \mathrm{~mm}$ de diámetro y longitud, respectivamente. El fruto cosechado en la etapa de madurez 3 se clasificó como "medio", con un peso promedio de 4,46 \pm $1,22 \mathrm{~g}$ y un diámetro y longitud de 18,9 $\pm 0,23$ y 23,5 $\pm 0,28 \mathrm{~mm}$. Además, se evidenció que a mayor calibre mayor peso, esto concuerda con lo descrito por Westwood (1982), quien indicó que el calibre (diámetro y longitud) de la fruta está estrechamente relacionado con el peso de la fruta.
El test de Tukey al 5\% identificó diferencias significativas en los pesos de la fruta (tabla $3)$, los valores numéricos son similares a lo descrito por Sánchez et al., (2018), quienes estudiaron en tres zonas agroclimáticas, alto de 2500 a 3000 m.s.n.m, medio con altitud superior a 2100 e inferior a 2500 m.s.n.m y bajo con altitud inferior a 2100 m.s.n.m y determinaron que no existió diferencias estadistas del peso del fruto en las altitudes evaluadas, con valores para la zona alta de 6,25 , zona media de 5,79 y zona baja con 6,53 gramos correspondientes en su mayoría a calibre pequeño. Según Franco et al., (1996), las bajas temperaturas hacen que los frutos sean más pequeños. Los pisos térmicos que se encuentran entre los 10 a $12{ }^{\circ} \mathrm{C}$, el fruto tiende a ser de tamaño pequeño, ya que no existen las suficientes unidades de calor que permitan su mayor desarrollo (Eraso, 1988).

TABLA III. CALIBRE Y PESOS DE LOS FRUTOS DE MORA

\begin{tabular}{|c|c|c|c|c|}
\hline $\begin{array}{c}\text { Zona } \\
\text { productora }\end{array}$ & Calibre & $\begin{array}{l}\text { Peso } \\
\text { (g) }\end{array}$ & $\begin{array}{c}\text { Frecuencia } \\
\text { absoluta }\left(\mathbf{n}_{\mathbf{i}}\right)\end{array}$ & $\begin{array}{c}\text { Frecuencia } \\
\text { relativa \% }\left(f_{i}\right)\end{array}$ \\
\hline \multirow{4}{*}{ Guantug Cruz } & Pequeño & $2-6 a$ & 425 & 94,44 \\
\hline & Mediano & $7-8 \mathrm{~b}$ & 24 & 5,33 \\
\hline & Grande & $9-10 \mathrm{c}$ & 1 & 0,22 \\
\hline & & Total & 450 & $100 \%$ \\
\hline \multirow{4}{*}{ Guarumal } & Pequeño & $2-6 a$ & 403 & 89,56 \\
\hline & Mediano & $7-8 b$ & 44 & 9,78 \\
\hline & Grande & $9-10 \mathrm{c}$ & 3 & 0.67 \\
\hline & & Total & 450 & $100 \%$ \\
\hline \multirow{4}{*}{ Tambo Real } & Pequeño & $2-6 a$ & 337 & 90,35 \\
\hline & Mediano & $7-8 b$ & 32 & 8,58 \\
\hline & Grande & $9-10 \mathrm{c}$ & 4 & 1,07 \\
\hline & & Total & 373 & $100 \%$ \\
\hline \multirow{4}{*}{ La Palma } & Pequeño & $2-6 a$ & 247 & 77,19 \\
\hline & Mediano & $7-8 \mathrm{~b}$ & 52 & 16,25 \\
\hline & Grande & $9-13 \mathrm{c}$ & 21 & 6,56 \\
\hline & & Total & 320 & $100 \%$ \\
\hline
\end{tabular}

Medias con una letra común no son significativamente diferentes $(p>0.05)$.

Fuente: Los autores 


\section{B. Rendimiento de la pulpa}

La Tabla 4 muestra que el rendimiento de pulpa fue mayor de la mora proveniente de Guantug Cruz seguido por Guarumal, con el $71,48 \%$ y $71,30 \%$ respectivamente, el calibre de los frutos no estuvo relacionado con la eficiencia de extracción, porque la mayoría de los frutos fueron de calibre pequeño (peso menor o igual a $6 \mathrm{~g}$ ) 94,23\% y 89,56 $\%$ respectivamente. Al respecto Montalvo (2012), en su investigación determinó que el rendimiento de pulpa no fue la de mayor peso de la fruta.

El rendimiento de la pulpa está relacionado con la cantidad de sólidos solubles totales (SST) presentes en el fruto como azúcares, ácidos, sales y demás compuestos solubles en agua presentes en los jugos de las células de una fruta, por lo tanto, las muestras que presentan valores superiores son adecuadas para ser procesadas.

Un estudio realizado por Esparza et al., (2004) determinó que el rendimiento de la pulpa de mora mínimo requerido es de un $80 \%$, los resultados de este estudio son inferiores, García y García, (2001), señalan que la calidad y el valor nutritivo de la mora, están influenciados por cambios físicos $\mathrm{y}$ químicos que ocurren durante su maduración, conservación y posterior procesamiento.
TABLA IV. RENDIMIENTO DE LA PULPA DE FRUTOS DE MORA

\begin{tabular}{cccc}
\hline $\begin{array}{c}\text { Zona } \\
\text { productora }\end{array}$ & $\begin{array}{c}\text { Peso fruta } \\
(\mathbf{g})\end{array}$ & $\begin{array}{c}\text { Peso pulpa } \\
(\mathbf{g})\end{array}$ & $\begin{array}{c}\text { Rendimiento } \\
(\%)\end{array}$ \\
\hline Guantug Cruz & 7370 & 5268 & 71,48 \\
Guarumal & 4637 & 3306 & 71,30 \\
Tambo Real & 1858 & 1245 & 67,01 \\
La Palma & 1671 & 914 & 54,70 \\
\hline
\end{tabular}

Fuente: Los autores

\section{Estado de madurez}

En la Tabla 5, se registraron los estados de madurez de los frutos recolectados. Los estados de madurez 3, 4 y 6 en las zonas evaluadas presentan los valores más altos ( $\square=50,17 \%$ ). Sin embargo, las zonas que presentan valores acordes a la normativa (estado de madurez 5) son Tambo Real y La Palma con $61,93 \%$ y 54,06\% respectivamente. Los estados de madurez 5 (Fruto de color rojo intenso, con algunas drupas de color morado), presentan condiciones de madurez aptas para la comercialización y consumo humano; mientras que el estado de madurez 6 (fruto de color morado oscuro), equivale el $46,70 \%$ del total de las muestras, demostrando ser un problema en el manejo del producto, por su pérdida de turgencia y humedad excesiva que generan procesos de fermentación y proliferación de hongos, convirtiéndolo en un producto de rechazo (Tabla 5 y figura 2). 
TABLA V. TABLA DE FRECUENCIAS DE LOS ESTADOS DE MADUREZ DE MORA

\begin{tabular}{|c|c|c|c|}
\hline $\begin{array}{c}\text { Zona } \\
\text { productora }\end{array}$ & $\begin{array}{c}\text { Estado de } \\
\text { madurez }\end{array}$ & $\begin{array}{l}\text { Frecuencia absoluta } \\
\left.\text { ( } \mathrm{n}_{\mathrm{i}}\right) \text { (Número frutos) }\end{array}$ & $\begin{array}{c}\text { Frecuencia } \\
\text { relativa \% }\left(\mathrm{f}_{\mathrm{i}}\right)\end{array}$ \\
\hline \multirow{5}{*}{ Guantug Cruz } & 3 & 3 & 0,67 \\
\hline & 4 & 62 & 13,78 \\
\hline & 5 & 240 & 53,33 \\
\hline & 6 & 145 & 32,22 \\
\hline & Total & 450 & 100 \\
\hline \multirow{4}{*}{ Guarumal } & 4 & 3 & 0,67 \\
\hline & 5 & 135 & 30,00 \\
\hline & 6 & 312 & 69,33 \\
\hline & Total & 450 & 100 \\
\hline \multirow{3}{*}{ Tambo Real } & 5 & 231 & 61,93 \\
\hline & 6 & 142 & 38,07 \\
\hline & Total & 373 & 100 \\
\hline \multirow{4}{*}{ La Palma } & 4 & 2 & 0,63 \\
\hline & 5 & 173 & 54,06 \\
\hline & 6 & 145 & 45,31 \\
\hline & Total & 320 & 100 \\
\hline
\end{tabular}

Fuente: Los autores

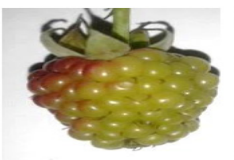

Estado de

madurez $O$

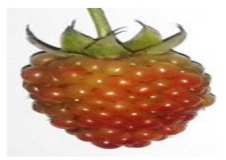

Estado de madurez 1

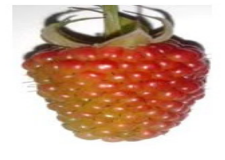

Estado de
madurez 2

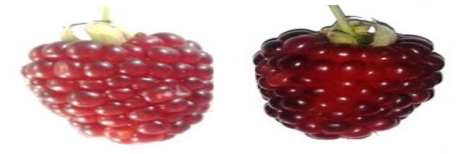

Estado de Estado de madurez 3 madurez 4

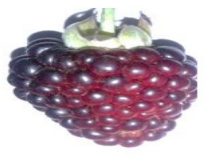

Estado de madurez 5

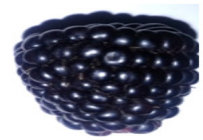

Estado de madurez 6

Fig. 2. Estados de madurez de acuerdo a su color de la mora de castilla.

De acuerdo a la Norma de Calidad Ecuatoriana NTE-2427 (INEN, 2010), la cosecha puede realizarse una vez que la fruta alcanza, como mínimo, la etapa de madurez 3, definida como roja clara. Sin embargo, Ayala-Sánchez et al. (2013), concluyeron que las bayas deberían cosecharse en estado de madurez 5, en esta etapa, la fruta presenta un tamaño adecuado, forma característica, ácidos apropiados y contenido de sólidos solubles totales.

\subsection{Análisis físico-químico}

Los resultados muestran que los frutos de la mora son ácidos (tabla 6). Los valores encontrados están dentro del rango obtenido por Farinango, (2010), quien reportó un valor de 2,79+/- 0,10 para la mora de castilla, así también Barrero, (2009) encontró valores en un rango de $\mathrm{pH}$ de 2,28 a 2,71.

La cantidad de sólidos solubles presentes en las muestras de mora son diferentes debido a que la cosecha se realizó en la época de invierno y en pisos altitudinales diferentes. Al respecto Sánchez et al, (2018), encontró diferencias en cuanto al contenido de sólidos solubles en los frutos de mora por efecto de los pisos altitudinales donde se producen. Tabla 6.

Pinzón et al., (2007) señala que la acumulación de azúcares depende de la variedad y la relación fuente sumidero. Según Farinango, (2010) la mora de Castilla llega a valores de $11,30^{\circ}$ Brix y en otros países, en 
estudios de selección realizados en Colombia según Barrero, (2009) las mejores accesiones presentaron valores entre 6,6 y $8,2{ }^{\circ}$ Brix.

El valor de acidez titulable más alto se encontró en la mora proveniente de Guarumal con $3,02 \%$ y el valor más bajo es de Guantug Cruz con 2,56\%. Tabla 6. La acidez de los frutos disminuye a medida que la cantidad de sólidos solubles aumenta, se establecen valores de aceptación entre $2,5-3,0 \%$ de ácido cítrico. En cuanto a acidez titulable Cáceres y Corro (2017) determinaron que el contenido de ácido cítrico fue de $2,03 \%$ y $1,95 \%$.

El contenido de sólidos solubles y el porcentaje de acidez mencionados son características deseables que hacen al fruto más atractivo para el consumo, pues su acidez disminuye (Pinzón et al., 2007).

Los índices de madurez son criterios $\mathrm{o}$ parámetros que se emplean para determinar el grado de desarrollo de las frutas, la importancia depende de la fruta y su uso, un índice de madurez debe ser simple, económico, objetivo y preferentemente no destructivo (García y García, 2001). La cantidad de sólidos solubles aumentan con el proceso de maduración y la acidez disminuye, en este caso el índice de madurez es bajo debido a las condiciones ambientales poco favorables para alcanzar la cantidad de sólidos solubles que permitan incrementar su índice de madurez a pesar que la mayoría de la fruta fue cosechada en estados de madurez 5 y 6 . Tabla 6 .
TABLA VI. CARACTERÍSTICAS FISICOQUÍMICAS DE LA PULPA DE MORA

\begin{tabular}{ccccc}
\hline $\begin{array}{c}\text { Zona } \\
\text { productora }\end{array}$ & $\mathrm{pH}$ & ${ }^{\circ}$ Brix & $\begin{array}{c}\text { Acidez Titulable } \\
\text { (\% Ácido Cítrico) }\end{array}$ & $\begin{array}{c}\text { Índice de } \\
\text { Madurez }\end{array}$ \\
\hline Guantug Cruz & 2,79 & 7,6 & 2,56 & 2,97 \\
Guarumal & 2,78 & 7,1 & 3,02 & 2,35 \\
Tambo Real & 2,77 & 7,4 & 2,73 & 2,71 \\
La Palma & 2,66 & 6,5 & 2,67 & 2,43 \\
\hline Promedio & 2,8 & 7,2 & 2,7 & 2,6 \\
\hline
\end{tabular}

Fuente: Los autores

\section{CONCLUSIONES}

El estado de madurez y el calibre de fruto son parámetros fundamentales que sirven de orientación para la cosecha y la comercialización, se evidenció que existió diferencias significativas en los pesos de los diferentes calibres en cada zona de estudio.

El rendimiento de la pulpa no dependió del calibre de la fruta, por lo tanto, los calibres pequeño, mediano y grande pueden ser utilizados para su procesamiento.

Los atributos de calidad como $\mathrm{pH}$, sólidos solubles, acidez titulable e índice de madurez son inferiores a los que establece la Norma de Calidad Ecuatoriana para su industrialización. 


\section{REFERENCIAS BIBLIOGRÁFICAS}

AOAC. (2007): Métodos oficiales de análisis de AOAC internacional, $18^{\mathrm{a}}$ edición, Maryland, Estados Unidos.

Ayala, L., C. Valenzuela, y Y. Bohórquez, (2013): "Caracterización fisicoquímica de mora de castilla (Rubus glaucus Benth) en seis estados de madurez". Biotecnología en el Sector Agropecuario y Agroindustrial, 11(2), 10-18.

Barrera, V., J. Alwang, G. Andrango, J. Domínguez, L. Escudero, A. Martínez, R. Jácome, y J. Arévalo, (2017): La Cadena de valor de la mora y sus impactos en la Región Andina del Ecuador. INIAP. Boletín Técnico No. 171. ARCOIRIS Producciones Gráficas. Quito, Ecuador.

Barrero, L. (2009): Caracterización, evaluación y producción de material limpio de mora con alto valor agregado. Publicado por CORPOICA, Cundinamarca, Colombia. Disponible en: https://repository.agrosavia.co/ handle/20.500.12324/12870

Bridgers, E.N., M.S. Chinn, y V.D. Truong, (2010): Extracción de antocianinas de camotes industriales de pulpa púrpura e hidrólisis enzimática de residuos para azúcares fermentables". Industrial Crops and Products 32, 613-620.

Cáceres, S.\&F.Corro,(2017): Caracterización agromorfológica y productiva de mora (Rubus glaucus Benth) en tres zonas agroecológicas de la provincia Bolívar (tesis de pregrado), Universidad Estatal de Bolívar. Guaranda-Ecuador.

Eraso, B. (1988): El cultivo de la mora en Colombia. En: Salazar, R. Memorias, Curso Nacional Sobre Frutales de Clima Frío. Volumen III, Técnicas de cultivo. Medellín. 42 p.

Esparza, E., X. Paladines, \& H. Arias, (2004): Evaluación económica, financiera $\mathrm{y}$ social de la instalación de una planta procesadora de pulpa de mora y tomate de árbol (tesis de pregrado). Escuela Superior Politécnica del Litoral, Guayaquil- Ecuador.

Farinango, M. (2010): Estudio de la fisiología poscosecha de la mora de castilla (Rubus glaucus Benth) y de la mora variedad brazos (Rubus sp.), (Tesis de pregrado), Ingeniería Agroindustrial. Quito (Ecuador): Escuela Politécnica Nacional, Facultad de Ingeniería Química y Agroindustria Quito, Ecuador.

Franco G., J. Bernal, J. Gallego, J. Rodríguez, N. Guevara, M. Londoño. (1996): Agronomía del Cultivo de Mora. En: Memorias Primer Seminario de Frutales de Clima Frio Moderado. Manizales, $1-19$.

Franco, G., M. Giraldo, (1999): El cultivo de mora. Primera edición. Corporación Colombiana de Investigación Agraria. 
$130 \mathrm{pp}$.

Freire, V. (2012): Alternativas de mejora en el manejo poscosecha y comercialización de la mora de castilla (Rubus glaucus Benth) proveniente de la provincia de Tungurahua (tesis de pregrado). Escuela Politécnica Nacional. QuitoEcuador.

García, M. y H. García, (2001): Manejo Cosecha y Postcosecha de mora, lulo y tomate de árbol, CORPOICA, primera edición, Bogotá-Colombia. 99pp.

Horvitz, S., D. Chanaguano, y I. Arozarena, (2017): "La calidad de las moras andinas (Rubus glaucus Benth) se ve afectada por la madurez de la cosecha y las condiciones de almacenamiento". Scientia Horticulturae, 226, 293-301. doi: 10.1016 / j.scienta.2017.09.002

INEN, (2010): Norma Técnica Ecuatoriana. NTE INEN 2427: 2010. Quito. Disponible en: https://archive.org/ stream/ec.nte.2427.2010\#page/n0/ mode/2up

INEN, (2015): Norma Técnica Ecuatoriana. Frutas frescas. Mora. Requisitos. NTE INEN 2427: 2010. Quito. Disponible en: http://www.normalizacion.gob.ec/wpcontent/uploads/downloads/2015/10/ nte_inen_2427_1r.pdf

Junqueira-Gonçalves, M.P., E. Alarcón, y K. Niranjan, (2016): "La eficacia del envasado recubierto de sorbato de potasio para controlar el moho gris de postcosecha en frambuesas, moras $\mathrm{y}$ arándanos". Postharvest Biology and Technology. 111, 205-208.

Martínez, A., O. Beltrán, G. Velastegui, G. Ayala, R. Jácome, W. Yánez, y E. Luciano, (2007). Manual del cultivo de la mora de castilla. Convenio INIAP - UTA, Ambato-Ecuador, Primera Edición.

Mertz, C., V. Cheynier, Z. Günata, y P. Brat, (2007): “Análisis de compuestos fenólicos en dos especies de mora (Rubus glaucus y Rubus adenotrichus) mediante cromatografía líquida de alta resolución con detección de matriz de diodos y espectrometría de masas con trampa de iones electrospray", Journal of Agricultural and Food Chemistry, 55(21), 8616-8624.

Meyers, S.L., K.M. Jennings, D.W. Monks, y W.E. Mitchem, (2014): "Efecto del ancho de la tira libre de malezas en el crecimiento de mora 'Navaho' recientemente establecido, el rendimiento y la calidad del fruto". Weed Technology, 28, 426-431.

Morales, A. L., D. Albarracin, J.Rodríguez, C. Duque, L. E. Riano, y J. Espitia, (1996). Componentes volátiles de la baya de los Andes (Rubus glaucus Benth). Journal of High Resolution Chromatography, 19, 585-587. 
Montalvo, D. (2012): Alternativas de mejora en el manejo poscosecha $\mathrm{y}$ comercialización de la mora de castilla (Rubus glaucus Benth) proveniente de la provincia de Tungurahua (tesis de pregrado). Escuela Politécnica Nacional. Quito. Ecuador. Disponible en: http://bibdigital.epn.edu.ec/ handle/15000/4609

Moreno-Medina, B.L., F. Casierra-Posada, and M. Blanke, (2016): "Índices de crecimiento en plantas de mora (Rubus alpinus Macfad) bajo diferentes sistemas de poda". Revista Colombiana de Ciencias Hortícolas, 10 (1), 28-39.

PAVUC, (2007): La mora andina (Rubus spp.) Hoja de datos $\mathrm{N}^{\circ}$ 3. Publicado por el Centro de Cultivos Subutilizados. Universidad de Southampton. Producir valor agregado de cultivos de frutas tropicales subutilizados con alto potencial comercial-PAVUC. Disponible en: https://www.pavuc. soton.ac.uk/fruits/fruits_factsheets/ Factsheet_3_rubus_final_3006.pdf

Perkins-Veazie, P., J. Collins, y J. Clark, (1999): "Período de validez y calidad de las frutas de zarzamora 'Navaho' y 'Shawnee' almacenadas en condiciones de almacenamiento minorista". Journal Food Quality, 22, 535-544.

Pinzón, I., F. Gerhard, y G. Corredor, (2007): "Determinación de los estados de madurez del fruto de gulupa (Passiflora edulis Sims)", Agronomía Colombiana,
$25(1), 83-95$.

Saltos, R. D. (Noviembre, 2019):“Caracterización morfológica cualitativa de ecotipos de mora (Rubus spp.) en tres localidades". Trabajo presentado la cuadragésima tercera edición de las Jornadas Nacionales de Biología, en el campus de la Universidad de Investigación de Tecnología Experimental Yachay.

Sánchez-Morales, J., M. Villares-Jibaja, Z. Niño-Ruiz, y M. Ruilova. (2018): "Efecto del piso altitudinal sobre la calidad de la mora (Rubus glaucus benth) en la región interandina del Ecuador". Revista IDESIA, 36 (2), 209215. DOI: http://dx.doi.org/10.4067/ S0718-34292018005000702

Skrovankova, S., D. Sumczynski, J. Mlcek, T. Jurikova, y J. Sochor, (2015): "Compuestos bioactivos y actividad antioxidante en diferentes tipos de bayas". International Journal of Molecular Sciences, 16, 24673-24706.

Sokal, R. R. \& F. J. Rohlf. (2012): Biometry. The Principles and Practice of Statistics in Biological Research". $4^{\text {th }}$ edition. W. H. Freeman and Company, New York. $937 \mathrm{pp}$

Sora, A., G. Fischer, y R. Flórez, (2006): "Almacenamiento refrigerado de frutas mora de Castilla (Rubus glaucus Benth.) En envases de atmósfera modificada". Agronomía Colombiana. 24, 306-316. 
Strik, B., J. Clark, C. Finn, y M. Bañados, (2008): "Producción mundial de moras". Acta Horticulturae, 777, 209218.

Strik, B., J. Clark, C. Finn, y P. Bañados, (2007): "Producción mundial de moras". Horticulture Technology, 17, 205-213.

Türemis, N., S. Kafkas, E. Kafkas, y C. Onur, (2003): "Características de la fruta de nueve genotipos de zarzamora sin espinas. Mermelada". Journal of the American Pomological Society, 57 (4), 161-165.

Universidad de Antioquia, (2009): Mora de castilla - Mora andina - Rubus glaucus Benth. Banco de Objetos de Aprendizaje y de Información. Disponible en http://aprendeenlinea. udea.edu.co/ova/?q=node/519

Vasco, C., J. Ruales, A. Kamal-Eldin, (2008): “Total de compuestos fenólicos y capacidades antioxidantes de las principales frutas de Ecuador". Food Chemistry, 111 (4), 816-823.

Westwood, N. (1982): Fruticultura de zonas templadas. Ediciones Mundi Prensa, Madrid. 461 pp.

Zar, J. H. (2010): Biostetistical Analysis. 5th edition. Pearson Education, Inc. Upper Saddle River, New Jersey. 944 pp. 